\title{
Effect of solid-solid phase transformations on elastic constitutive relations
}

\author{
T. Lookman, T.O. Williams and A. Saxena
}

Theoretical Division, Los Alamos National Laboratory, Los Alamos, New Mexico 87545, U.S.A.

\begin{abstract}
A two-dimensional (2D) Ginzburg-Landau (GL) theory for temperature induced phase transformations in single crystals is extended to incorporate the effects of mechanical and thermal (expansion or dilatational) strain effects. This extension of the GL theory allows the single crystal GL model to be implemented into polycrystalline homogenization schemes. The proposed model is capable of examining the interactions of applied mechanical loading states and phase transformation processes within single crystals.
\end{abstract}

\section{INTRODUCTION}

The objective of this work is to model the influence of solid-solid phase transformations (SSPT) at the single crystal level on the effective/bulk behavior of polycrystalline metals. Solid-solid phase transformations ${ }^{1}$ occur in many metals (such as actinides, steels, shape memory alloys, etc.). These transformations result in changed crystalline structures from that of the parent phase in microscopic regions within a single crystal. The SSPT processes proceed as a function of the bulk temperature, bulk stress, and time. The resulting changes in the single crystal atomic structure lead to nontrivial changes in the overall/bulk behavior of the transforming polycrystal/metal. In order to use metals that undergo SSPT in structural applications it is necessary to have appropriate constitutive models that account for the effects of SSPT on the overall response of the material.

We aim to develop the necessary constitutive models for metals undergoing SSPT by focusing on the evolution of solid-solid phase transformations in single crystals and polycrystals as a function of macroscopic stress, temperature, and loading history. The methodology consists of a theoretical, multilength scale bridging framework obtained by combining a modified Ginzburg-Landau theory ${ }^{2}$ for the evolution of solid-solid phase transformations in single crystals with homogenization based models ${ }^{3}$. The resulting theory simultaneously bridges phenomena occurring over four different length scales ranging from the atomistic to the macroscopic.

The Ginzburg-Landau (GL) formalism represents the bridging theory from the atomic to the single crystal levels. Ginzburg-Landau theory is based on the identification of fundamental deformation modes of the parent crystalline structures within a single crystal. Some of these deformation modes represent the phase transformations. The remaining modes represent accommodation effects required to maintain a continuum. These fundamental fields are related in a nonlocal manner through the use of the compatibility relations of geometrically linear elasticity ${ }^{4}$. We have extended the GL formalism in a consistent fashion to incorporate the effects of mechanical loading and thermal dilatation effects on the single crystal behavior. We envision that future work on the GL theory will incorporate the effects of plasticity on the SSPT behavior within single crystals. A typical microstructure predicted by the GL theory in a single crystal subjected to purely thermal loading is given in Fig. 1 along with how it provides input to the micromechanical modeling (discussed below, Fig. 2).

Next we embed the GL theory in homogenization models. Homogenization theory bridges the single crystal to macroscopic length scales. The particular homogenization model chosen here is a recently developed fully coupled thermo-mechanical theory ${ }^{3}$. This homogenization model assumes the existence of a deterministic polycrystalline structure and initially models each crystal in the polycrystal as a distinct subregion (see Fig. 2). The homogenization analysis satisfies the governing equations of linear continuum 
mechanics (equilibrium and energy) in an integral sense within each single crystal in the polycrystalline microstructure. The responses within the different crytals are coupled through the interfacial constraints on displacements and stresses and through the assumptions of periodicity. The theory is capable of analyzing general microstructures and is thus capable of being used to study localization effects within a polycrystalline structure.

The resulting coupled GL/homogenization theory provides the tools necessary to study how SSPT processes ocicur within individual crystals within a polycrystal, how the single crystals interact with each other, and how all of these processes impact the bulk behavior of the metal as a function of bulk thermomechanical loading history. The theoretical framework will allow the influence of solid-solid phase transformations on the overall/effective response of metals during casting processes, machining processes, and static and dynamic loading of complex structures to be predicted. In addition, the resulting theory will provide insight into the necessary functional forms that phenomenological constitutive models must have in order to correctly model the influence of solid-solid phase transformations. The resulting derived phenomenological models can subsequently be used in large structural simulations in a computationally efficient manner.

\section{GINZBURG-LANDAU MODELING}

In a $2 \mathrm{D}$ theory the phase transformation process of interest corresponds to a square to rectangular deformation mode. The associated order parameter (OP) used to model the phase transformation is a deviatoric strain effect. This OP for the transformation process is required to vanish in the pure high temperature square phase.

To illustrate the above points we first consider only the Ginzburg-Landau part of the modeling in 2D, namely a square to rectangle transformation. Our model ${ }^{5}$ consists of (i) a triple-well potential $F_{0}$, as is usual for first order transitions, in the deviatoric strain oP $\left[\varepsilon=(1 / \sqrt{2})\left(\varepsilon_{\mathrm{xx}}-\varepsilon_{\mathrm{yy}}\right)\right]$, (ii) harmonic (linear) elastic energy cost $F_{\text {cs }}$ due to the compressional $\left[\mathrm{e}_{1}=(1 / \sqrt{2})\left(\varepsilon_{\mathrm{xx}}+\varepsilon_{\mathrm{yy}}\right)\right]$ and shear $\left(\mathrm{e}_{2}=\varepsilon_{\mathrm{xy}}\right)$ strain that implicitly depends on the OP through the elatic compatibility constraint; (iii) coupling of strain(s) to an external or internal (defect, dislocation) stress, and (iv) second order strain gradient terms $F_{\text {grad }}$ that induce multiscale competition. For a specific material the coefficients in the GL model can be determined from the measured structural and phonon dispersion data.

There is a connection between compressional, shear and rectangular strains, as they are different components of the strain tensor, i.e. are derivatives of the same underlying displacement field $\vec{u}(R)$. The OP $\varepsilon(R)$ acts as a source term in the compatibility equation, inducing variations in $e_{1}(R), e_{2}(R)$. The $2 D$ compatibility constraint ${ }^{5}$, is $\nabla^{2} \mathrm{e}_{1}(\mathrm{R})-\sqrt{8} \nabla_{\mathrm{x}} \nabla_{\mathrm{y}} \mathrm{e}_{2}(\mathrm{R})=\left(\nabla_{\mathrm{x}}^{2}-\nabla_{\mathrm{y}}^{2}\right) \varepsilon(\mathrm{R})$. The compression-shear (CS) strains $e_{1}(R), e_{2}(R)$ are written in terms of the order parameter $\varepsilon(R)$ by solving the elastic compatibility (differential) equation ${ }^{4}$. Expressing $F_{\mathrm{cs}}$ is equivalent to inducing an anisotropic, long-range interaction, of the form $U(\vec{R}-\vec{R}) \sim \cos \left[4\left(\theta-\theta^{\prime}\right)\right] /\left(\vec{R}-\vec{R}^{\prime}\right)^{2}$. This interaction is responsible for microstructure such as twinning.

The dynamics of the continuous $O P$ is assumed to be of the time dependent GL or relaxational type, $\dot{\varepsilon}(R)=-\partial F\left(\left\{(R), e_{1}(\varepsilon(R)), e_{2}(\varepsilon(R))\right\} / \partial \varepsilon(R)\right.$, where time $t$ is scaled by a characteristic relaxation time. A typical GL microstructure is depicted in Fig. 1.

\section{INCLUSION OF MECHANICAL STRAINS}

The GL formalism represents the kinematic behavior of a metal, or more generally a material, in terms of fundamental, independent deformation modes of the crystalline lattice structure. For the currently proposed $2 \mathrm{D}$ theory these fundamental modes are characterized by a deviatoric (or rectangular), a dilatation and a shear strain, respectively defined by strain $\varepsilon^{T}=\frac{1}{\sqrt{2}}\left(\varepsilon_{x x}-\varepsilon_{y y}\right), e_{1}^{T}=\frac{1}{\sqrt{2}}\left(\varepsilon_{x x}+\varepsilon_{y y}\right)$, and 
$\mathrm{e}_{2}^{\mathrm{T}}=\mathrm{e}_{\mathrm{xy}}$, where superscript $T$ denotes total strains. The corresponding stresses are given by $\sigma_{\varepsilon}=\frac{1}{\sqrt{2}}\left(\sigma_{x x}-\sigma_{y y}\right), \sigma_{1}=\frac{1}{\sqrt{2}}\left(\sigma_{x x}+\sigma_{y y}\right)$, and $\sigma_{2}=\sigma_{x y}$. For the $2 \mathrm{D}$ case the (martensitic) solid-solid phase transformation process is defined as a change from a square to a rectangular deformation mode. Thus, the order parameter used to distinguish the SSPT process in the theory is associated with the deviatoric strain defined above.

The above relations are based on the total strains at a point within the single crystal. It is assumed (for small strains, $<10 \%$ deformation) that the total strains are obtained from a superposition of the mechanical and transformation strain effects. $\varepsilon^{\mathrm{T}}=\varepsilon^{\mathrm{M}}+\varepsilon^{\mathrm{GL}}, \mathrm{e}_{1}^{\mathrm{T}}=\mathrm{e}_{1}^{\mathrm{M}}+\mathrm{e}_{1}^{\mathrm{GL}}$ and $\mathrm{e}_{2}^{\mathrm{T}}=\mathrm{e}_{2}^{\mathrm{M}}+\mathrm{e}_{2}^{\mathrm{GL}}$ where superscript $M$ denotes mechanical (and thermal expansion) effects and superscript $G L$ denotes the strain effects associated with SSPT processes. It is required that $\varepsilon^{\mathrm{GL}}=\mathrm{e}_{1}^{\mathrm{GL}}=\mathrm{e}_{2}^{\mathrm{GL}}=0$ in the pure square (or austenite) phase.

General Free Energy Expression: In terms of these strain effects a general expression for the (harmonic) free energy at a point within the single crystal is given by

$$
\begin{aligned}
& \mathcal{F}=\frac{1}{2} \mathrm{~A}_{1}^{\mathrm{M}} \mathrm{e}_{1}^{\mathrm{M}} \mathrm{e}_{1}^{\mathrm{M}}+\frac{1}{2} \mathrm{~A}_{2}^{\mathrm{M}} \mathrm{e}_{2}^{\mathrm{M}} \mathrm{e}_{2}^{\mathrm{M}}+\frac{1}{2} \mathrm{~A}_{\varepsilon}^{\mathrm{M}} \varepsilon^{\mathrm{M}} \varepsilon^{\mathrm{M}} \\
& +\frac{1}{2} \mathrm{~A}_{1}^{\mathrm{GL}} \mathrm{e}_{1}^{\mathrm{GL}} \mathrm{e}_{1}^{\mathrm{GL}}+\frac{1}{2} \mathrm{~A}_{2}^{\mathrm{GL}} \mathrm{e}_{2}^{\mathrm{GL}} \mathrm{e}_{2}^{\mathrm{GL}}+\frac{1}{2} \mathrm{~A}_{\varepsilon}^{\mathrm{GL}} \varepsilon^{\mathrm{GL}} \varepsilon^{\mathrm{GL}} \\
& +\frac{1}{2} \mathrm{~A}_{\varepsilon \mathrm{GL}}^{\mathrm{GL}} \mathrm{e}^{\mathrm{M}} \mathrm{e}^{\mathrm{GL}}+\frac{1}{2} \mathrm{~A}_{11}^{\mathrm{GL}} \mathrm{e}_{1}^{\mathrm{M}} \mathrm{e}_{1}^{\mathrm{GL}}+\frac{1}{2} \mathrm{~A}_{22}^{\mathrm{GL}} \varepsilon_{2}^{\mathrm{M}} \varepsilon_{2}^{\mathrm{GL}}
\end{aligned}
$$

The strains $\varepsilon^{\mathrm{GL}}, \mathrm{e}_{1}^{\mathrm{OL}}, \mathrm{e}_{2}^{\mathrm{GL}}$ are expressed in terms of the total and mechanical strains through compatibility. This free energy form can be considered to be a specialized form of a Helmholtz free energy. The free energy is related to the internal energy $\mathrm{u}$ by $u=F+T s$, where $T$ is the absolute temperature and $s$ is the entropy of the system. The SSPT processes are included by augmenting this energy expression with nonlinear terms in $\varepsilon^{G L}$. The individual coefficients in the free energy are related to the material elastic constants.

Thermo-Dynamics Constraints: Substitution of the above free energy form into the first law of thermo-dynamics $\rho \dot{u}=\underline{\underline{\sigma}}: \underline{\underline{\varepsilon}}-\underline{\nabla} \cdot \mathbf{q}$ and the Clausius-Duhem inequality (a particular form of the second law of thermo-dynamics) $\rho T \dot{s}+\nabla \cdot \underline{q}-\frac{q-\underline{q} T}{T} \geq 0$ results in constitutive and evolution constraints that need to be satisfied by the formulation. Here $\rho, \sigma$ and $q$ denote mass density, stress and amount of heat, respectively. In particular, the constraints on the mechanical behavior of the material relevant to the current analysis (obtained from the strong assumption that concerns satisfying the Clausius-Duhem inequality) are $\underline{\underline{\sigma}}^{\mathrm{M}}=\frac{\partial \mathrm{F}}{\partial \underline{\underline{\underline{\varepsilon}}}} \stackrel{\underline{\underline{\sigma}}}{\underline{\underline{\sigma L}}}=\frac{\partial \mathrm{F}}{\partial \underline{\underline{\varepsilon}}^{\mathrm{GL}}}$ and $-\frac{\partial F}{\partial \underline{\underline{\varepsilon}}}: \dot{\underline{\varepsilon}}^{\mathrm{GL}} \geq 0$.

Constitutive Relations: We embed the GL model into the micromechanical modeling according to the scheme in Fig. 2. The mechanical stresses $\sigma_{0}^{\mathrm{M}}, \sigma_{1}^{\mathrm{M}}, \sigma_{2}^{\mathrm{M}}$ are derived in terms of the mechanical strains and $\varepsilon^{\mathrm{GL}}$. The requirement of mechanical equilibrium on the stresses leads to two equations for the displacements $\left(u_{x}, u_{y}\right)$ in terms of $\varepsilon^{\mathrm{GL}}, \mathrm{e}_{1}^{\mathrm{GL}}, \mathrm{e}_{2}^{\mathrm{GL}}$. Thus, the overall algorithm consists of solving for $\varepsilon^{\mathrm{GL}}$ (and hence $\mathrm{e}_{1}^{\mathrm{GL}}, \mathrm{e}_{2}^{\mathrm{GL}}$ ) using the evolution equation from which the mechanical strains, total strains, and thus the stresses may be obtained. Different loading histories may be considered and to interface with micromechanical modeling, we can easily apply an average strain that is conserved in the course of the simulations. Imposing a constant average strain is like introducing a constant field. The equations are readily modified to incorporate this constraint. 


\section{CONCLUSION}

We have provided a preliminary account of a computational method that aims to capture the effect of microstructure on constitutive relations of (martensite like) materials undergoing a structural transformations. The resulting coupled GL/homogenization theory provides the tools necessary to study how SSPT processes occur within individual crystals in a polycrystal, how the single crystals interact with each other, and how all of these processes impact the bulk behavior of the metal as a function of bulk thermo-mechanical loading history. The theoretical framework will allow the influence of solid-solid phase transformations on the overall/effective response of metals (such as actinides, steels, shape memory alloys, etc.) during casting processes, machining processes, and static and dynamic loading of complex structures to be predicted. In addition, the resulting theory will provide insight into the necessary functional forms that phenomenological constitutive models must have in order to correctly model the influence of solid-solid phase transformations. The resulting derived phenomenological models can subsequently be used in large structural simulations in a computationally efficient manner.

This work was supported by the U. S. Department of Energy.

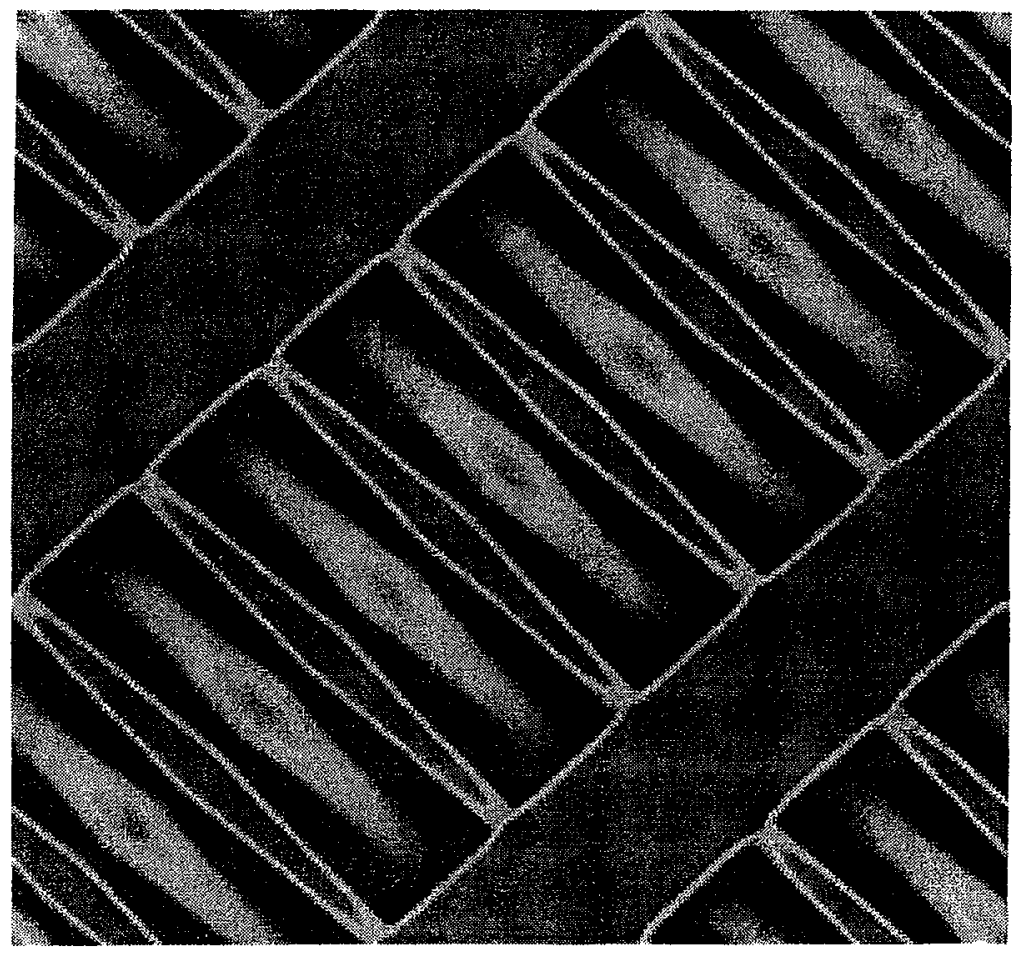

FIG. 1. A typical microstructure obtained from the 2D time-dependent GL simulations.

\footnotetext{
${ }^{1}$ A. D. Bruce and R. A. Cowley, Structural Phase Transitions (Taylor and Francis, London, 1981).

${ }^{2}$ J.-C. Toledano and P. Toledano, The Landau Theory of Phase Transitions (World Scientific, New Jersey, 1987).
}

${ }^{3}$ J. Aboudi, M. J. Pindera, and S. M. Arnold, Intern. J. Solids and Structures 31, 1393 (1994).

${ }^{4}$ D. S. Chandrasekharaiah and L. Debnath, Continuum Mechanics (Academic Press, San Diego, 1994).

${ }^{5}$ S.R. Shenoy, T. Lookman, A. Saxena, and A. ft. Bishop, Phys. Rev. B 60, R12537 (1999). 


\section{Homogenization}

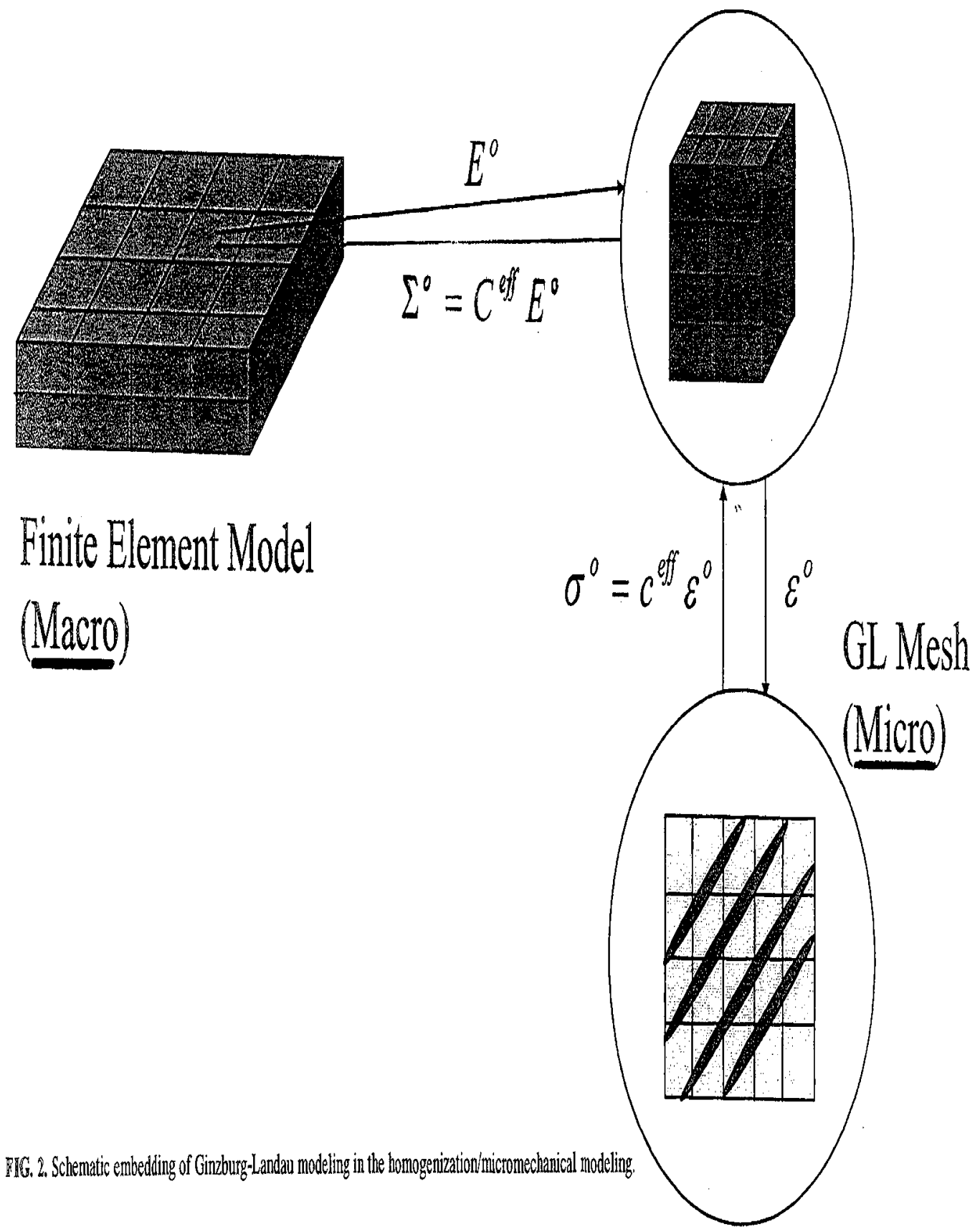

